\title{
High Precision Clock Bias Prediction Model in Clock Synchronization System
}

\author{
Zan Liu, ${ }^{1}$ Xihong Chen, ${ }^{1}$ Jin Liu, ${ }^{2}$ and Chenglong $\mathrm{Li}^{1}$ \\ ${ }^{1}$ Air and Missile Defense College, Air Force Engineering University, Xian 710051, China \\ ${ }^{2}$ Unit 95425 of the Chinese People's Liberation Army, Qujing 655000, China \\ Correspondence should be addressed to Zan Liu; kgdliuzan@163.com
}

Received 19 July 2016; Revised 18 October 2016; Accepted 23 October 2016

Academic Editor: Magdi S. Mahmoud

Copyright (c) 2016 Zan Liu et al. This is an open access article distributed under the Creative Commons Attribution License, which permits unrestricted use, distribution, and reproduction in any medium, provided the original work is properly cited.

\begin{abstract}
Time synchronization is a fundamental requirement for many services provided by a distributed system. Clock calibration through the time signal is the usual way to realize the synchronization among the clocks used in the distributed system. The interference to time signal transmission or equipment failures may bring about failure to synchronize the time. To solve this problem, a clock bias prediction module is paralleled in the clock calibration system. And for improving the precision of clock bias prediction, the firstorder grey model with one variable $\operatorname{GM}(1,1))$ model is proposed. In the traditional $\operatorname{GM}(1,1)$ model, the combination of parameters determined by least squares criterion is not optimal; therefore, the particle swarm optimization (PSO) is used to optimize GM(1, 1) model. At the same time, in order to avoid PSO getting stuck at local optimization and improve its efficiency, the mechanisms that double subgroups and nonlinear decreasing inertia weight are proposed. In order to test the precision of the improved model, we design clock calibration experiments, where time signal is transferred via radio and wired channel, respectively. The improved model is built on the basis of clock bias acquired in the experiments. The results show that the improved model is superior to other models both in precision and in stability. The precision of improved model increased by $66.4 \% \sim 76.7 \%$.
\end{abstract}

\section{Introduction}

Time synchronization technology has been widely used in the distributed system [1-4], such as global navigation satellite system (GNSS) and multistatic radar and electric network. Clock calibration is that devices of distributed system use reference time to synchronize the local clock. Hence, process of clock calibration determines the accuracy of time synchronization [5-7]. Nowadays, the way to calibrate local clock is that finite impulse response (FIR) filter uses the bias between local and remote clock measured by time interval counter (TIC) or discriminator to get a control signal [8-10], which is used to regulate clock until the clocks are synchronized. In the distributed system with far distance, time signal is always conveyed by electric cable, satellite, or microwave. Recently, troposphere scatter has also been proposed to transfer the time signal [6]. In a process of time signal's transmission, channel interruption or equipment failure will lead to the failure to get clock bias, and the absence of clock bias can make synchronization system abnormal.
In order to guarantee the distributed system work normally and add anti-interference ability of the synchronization system, clock bias prediction module is paralleled in the clock calibration system. In this parallel module, clock bias could be acquired through the prediction module, when the system cannot get clock bias. And system uses the predicted clock bias to generate a control signal, which is used to adjust the local clock. Above all, the performance of clock bias prediction has a direct impact on synchronization precision when accidents occur. At present, the clock bias prediction is an important work in GNSS; researchers have put forward several prediction models $[8,9]$, such as first-order grey model with one variable $(\operatorname{GM}(1,1))$ model, artificial neutral network (ANN) model, and least squares support vector machine (LSSVM) model. The sampling interval of satellite clock bias is generally $15 \mathrm{~min}$, which lead to a relatively low real-time requirement. Nevertheless, in the clock calibration system, the short sampling time puts forward a great requirement for real-time performance. $\operatorname{GM}(1,1)$ model needs less data sample and has better real-time than 


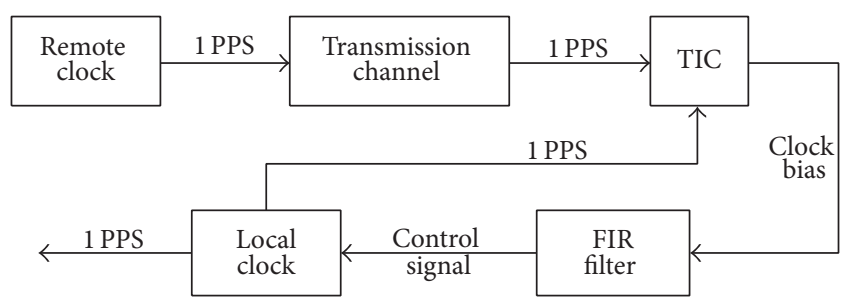

FIgURE 1: Workflow of clock calibration.

artificial intelligence algorithms, which need more data to train themselves. However, parameters of $\operatorname{GM}(1,1)$ model are usually determined by the least square criterion (LSC), which cannot guarantee the parameters are optimal.

Aiming at this problem, we introduce the particle swarm optimization (PSO) algorithm to optimize $\mathrm{GM}(1,1)$ model. Also, in order to avoid the PSO getting stuck at local optimization and improve its efficiency, the mechanisms that double subgroups and nonlinear decreasing inertia weight are proposed.

The rest of this paper is organized as follows. The mechanism that clock bias module is paralleled in clock calibration system is described in the next section. In Section 3, GM(1, 1) model optimized by improved particle swarm optimization (IPSO) is introduced. In Section 4, we design two clock calibration experiments that time signal is transferred via wired and radio channel, respectively. And the improved model is built through clock bias acquired by these calibration experiments. Finally, some conclusions are drawn in Section 5.

\section{Clock Bias Prediction in Clock Calibration}

Figure 1 shows the workflow of the clock calibration model in time synchronization system.

As shown in Figure 1, TIC is used to calculate the bias between these two one pulse per second (1 PPS), which come from the local and remote clock, respectively. FIR filter is used to generate a control signal on the basis of clock bias. Local clock continues to be adjusted according to the control signal until clocks are synchronous. Briefly, when the transmission channel or equipment is in a fault state, the local system cannot get the essential clock bias. Namely, absence of clock bias will bring a failure to time synchronization system. At present, no effective solutions exist or are proposed to overcome this failure. So to overcome this shortcoming, clock bias module is paralleled in clock calibration system; this mechanism is described in Figure 2.

In Figure 2, prediction module paralleled with the original system can predict clock bias, which may not be got when exceptions occur. Also, bias prediction module is paralleled with the original system, so under normal circumstances, this new module has no influence on the original system. Generally speaking, this parallel mechanism can ameliorate anti-interference ability and guarantee the synchronization system work normally. And the accuracy of clock bias prediction has great influence on the performance of time synchronization, when an exception happens. Therefore, an excellent clock bias prediction model is urgently needed.

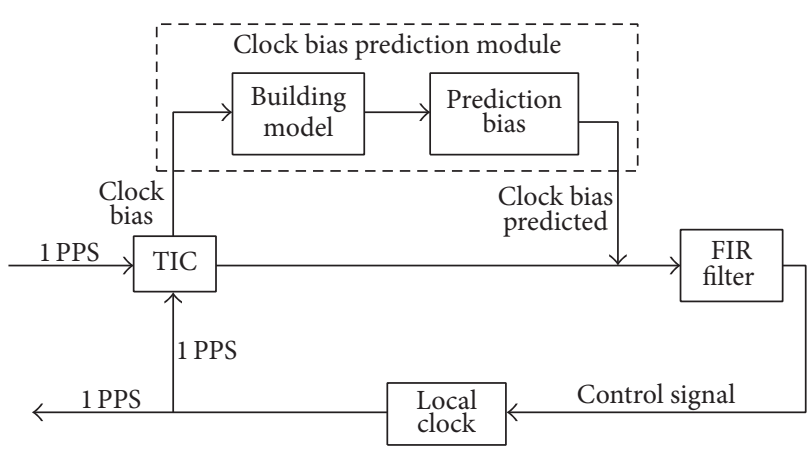

FIGURE 2: Clock error prediction in calibration.

\section{GM( $(1,1)$ Model Improved by IPSO}

3.1. $\operatorname{GM}(1,1)$ Model. The $\mathrm{GM}(1,1)$ model has advantage not only in calculation speed but also in prediction accuracy. Also, building $\operatorname{GM}(1,1)$ model needs less data [11-14]. $\operatorname{GM}(1,1)$ model can be finally expressed as

$$
\hat{x}^{0}(k)=e^{-a(k-1)} \cdot\left[x^{0}(1)-\frac{u}{a}\right] \cdot\left(1-e^{a}\right),
$$

where $x^{0}(k)$ and $\hat{x}^{0}(k)$ stand for the primitive and predicted $k$ th element in sequence, respectively. $a$ and $u$ are the parameters of this model. According to LSC, the estimated value of parameters can be expressed as

$$
[\widehat{a}, \widehat{u}]^{T}=\left(G^{T} G\right)^{-1} G^{T} Y_{n},
$$

where $G=[-Z(K+1), 1]_{(n-1) \times 2}, Z(K+1)=\left[x^{1}(k+1)+\right.$ $\left.x^{1}(k)\right] / 2$, and $Y_{n}=\left[x^{1}(2), \ldots, x^{1}(k), \ldots, x^{1}(n)\right]^{T} \cdot x^{1}(k)$ stand for the first accumulation of the primitive sequence. Equation (1) indicates that parameters directly affect the performance of $\operatorname{GM}(1,1)$ model. However, LSC cannot guarantee parameters are optimal.

For demonstrating that parameters obtained by LSC are not optimal, clock bias of satellite PG01 provided by the international GNSS service (IGS) in June 10 of 2012 is selected; the sampling interval is $15 \mathrm{~min}$. $\operatorname{GM}(1,1)$ models with different parameters are established by using these data during the first 18 hours. Data during last 6 hours are used to evaluate the performance of different models. Values of mean errors (ME) and mean square error (MSE) are used to evaluate accuracy of the model. MSE can be expressed as

$$
\operatorname{MSE}=\frac{1}{n} \sum_{i=1}^{n}\left(x_{i}-\widehat{x}_{i}\right)^{2},
$$

where $x_{i}$ and $\hat{x}_{i}$ stand for the real and predicted clock bias, respectively. As shown in (3), the minimum value of MSE refers to the optimal combination of parameters. There are two strategies to get different combinations of parameters. Strategy 1: combination of parameters is determined by LSC, shown as (2). Strategy 2: different combinations of parameters are chosen close to the parameters determined by LSC.

The values of ME and MSE are got through using different combinations of parameters. In these different models, the 


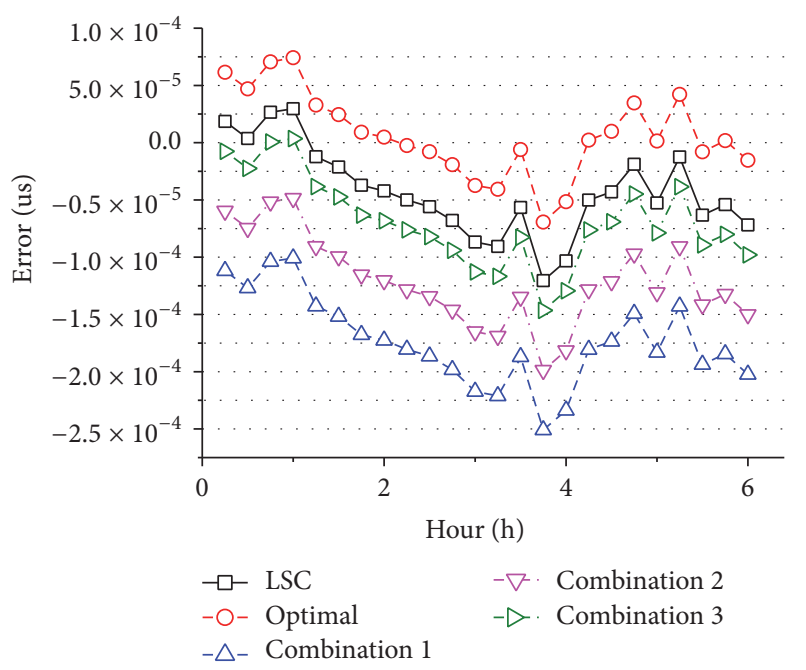

Figure 3: Prediction errors of different models.

optimal combination of $a$ and $u$ is $\left(-5.965 \times 10^{-6}, 260.74\right)$. And values of MSE and ME are $1.36 \times 10^{-3} \mathrm{~ns}^{2}$ and $0.0066 \mathrm{~ns}$, respectively. The combination of $a$ and $u$ determined by LSC is $\left(-5.963 \times 10^{-6}, 260.63\right)$. And the values of MSE and ME are $3.32 \times 10^{-3} \mathrm{~ns}^{2}$ and $-0.043 \mathrm{~ns}$, respectively. Prediction errors are shown as in Figure 3.

In Figure 3, the labels "combination 1," "combination 2," and "combination 3" stand for the different combinations close to parameters determined by LSC, respectively. And the label "optimal" stands for the optimal combination. As described in Figure 3, the parameters determined by LSC are not optimal. Therefore, using IPSO algorithm to optimize the $\operatorname{GM}(1,1)$ model is necessary.

3.2. IPSO Algorithm. PSO algorithm is widely used as a global optimization algorithm, which has the characteristics of simple programming, high efficiency, and fast computing speed [15-17]. The solution space is considered as a multidimension search space. Particle flying in the search space as an individual bird stands for a candidate solution. And the swarm is a collection of these particles. Candidate solution is judged by a fitness function, which usually depends on the problem to be optimized. During the process of optimization, each particle maintains a Pbest value as individual experience, which represents the best solution until now. Meanwhile, swarm maintains a Gbest value as social knowledge, which represents the best point achieved by the whole collection until now.

During iterative procedure, these particles fly through the problem space following the current optimum particle and corporate with each other to find the global optimum. The algorithm updates velocity and position of each particle as follows:

$$
\begin{aligned}
& v_{i d}^{k}=\omega v_{i d}^{k-1}+c_{1} r_{1}\left(p_{i d}-x_{i d}^{k-1}\right)+c_{2} r_{2}\left(g_{i d}-x_{i d}^{k-1}\right), \\
& x_{i d}^{k}=x_{i d}^{k-1}+v_{i d}^{k-1},
\end{aligned}
$$

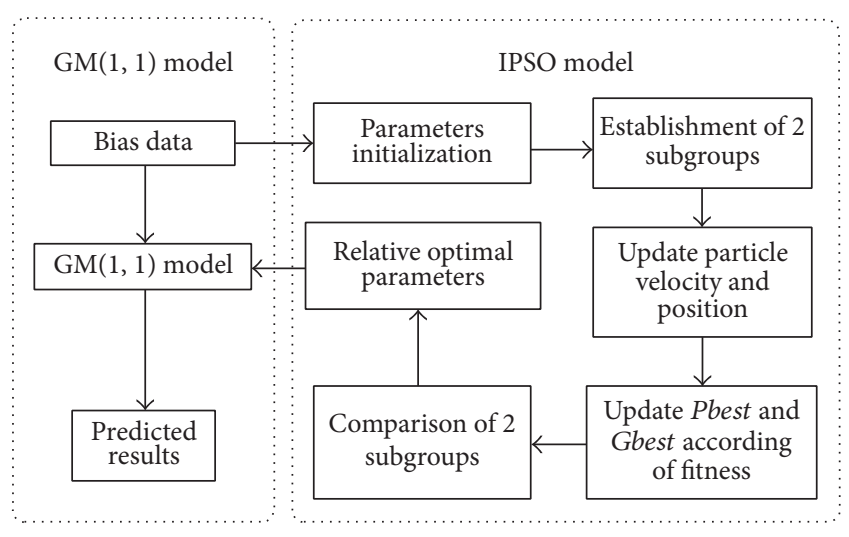

Figure 4: Flow of the improved model.

where $r_{1}$ and $r_{2}$ are random values between 0 and $1 . v_{i d}^{k}$ and $x_{i d}^{k}$ are velocity and position of particle $i$ in dimension $d$ at time $k$, respectively. $c_{1}$ and $c_{2}$ stand for acceleration constants known as the cognitive and social learning parameters, which pull each particle towards Pbest and Gbest positions, respectively. $\omega$ stands for the inertia weight reflecting the search ability of algorithm. Hence, in order to improve the accuracy and convergence rate of PSO, $\omega$ changing with the optimized iteration is proposed as follows:

$$
\omega_{k}=\left(\omega_{\max }-\omega_{\min }\right) \cdot e^{-\beta \cdot\left(k / k_{\max }\right)^{2}}+\omega_{\min },
$$

where $\omega_{\max }$ and $\omega_{\min }$ stand for the maximum and minimum of inertia weights, respectively. The value of $\beta$ is between 15 and 20. As described in (5), at the early optimization stage, a larger inertia weight factor is applied to promote global exploration; then, in order to facilitate local exploitation with algorithm running, it decreases in exponential form. At the same time, for avoiding PSO getting stuck at local optimization and improving its efficiency, the mechanism that double subgroups simultaneously search is proposed. On the basis of original algorithm, the backward searching subgroup is described as follows:

$$
x_{i d}(t+1)=x_{i d}(t)-v_{i d}(t+1) .
$$

After each cycle is complete, these two subgroups will compare with each other. This mechanism can make the algorithm avoid local optimization on the basis of not increasing the optimal algebra obviously.

3.3. GM(1,1) Model Improved by IPSO. In the time synchronization system, channel interruption or equipment failure will lead to a failure to get clock bias. Under these negative circumstances, clock bias acquired through a prediction model can guarantee the distributed system work normally. We use $\operatorname{GM}(1,1)$ model to predict these clock bias. In order to find the optimal parameters of $\operatorname{GM}(1,1)$ model, IPSO algorithm is proposed. The process of improved model is shown as in Figure 4.

As shown in Figure 4, GM $(1,1)$ model improved by IPSO mainly follows the basic steps of standard PSO. The detailed steps are as follows. 


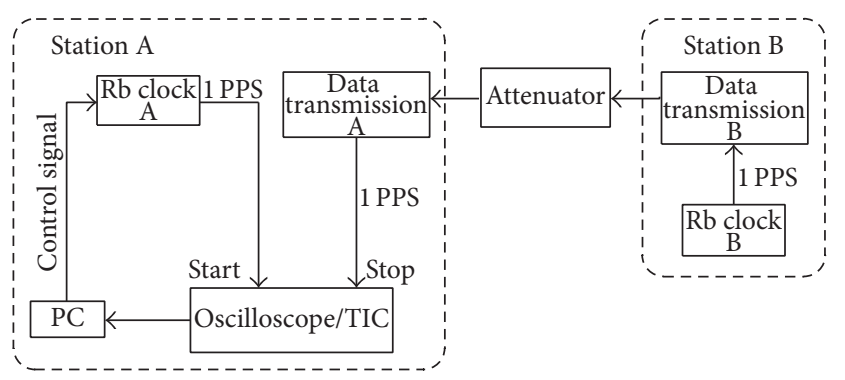

FIGURE 5: Clock calibration through wired channel.

Step 1. The parameters of IPSO are firstly initialized, including $\omega_{\min }\left(\omega_{\max }\right), c_{1}\left(c_{2}\right), \beta$, threshold of fitness, and the maximum number of circulating times.

Step 2. IPSO algorithm establishes two subgroups, which search in opposite direction according to (6).

Step 3. Velocity and position of particles are updated, and the Pbest and Gbest of particles are also updated according to the value of fitness.

Step 4. In order to find the optimal combination of parameters, fitness of two subgroups is compared.

Step 5. IPSO algorithm continues to find the relative optimal combination of parameters, until it reaches the maximum number of circulating times or the value of fitness meets the threshold.

Step 6. After IPSO algorithm finishes, $\operatorname{GM}(1,1)$ model with the relative optimal combination of parameters determined by IPSO is used to predict the clock bias.

\section{Example Analysis}

4.1. Clock Bias Acquisition Schemes. In order to evaluate the performance of $\operatorname{GM}(1,1)$ model improved by IPSO, clock bias acquisition experiment is conducted with PSR10type rubidium clock, Agilent53230A-type TIC, Tektronix DPO3054-type Oscilloscope, and so forth. The wired data transmission experiment is firstly performed as the layout shown in Figure 5.

As shown in Figure 5, 1 PPS produced by Rb clock B is transferred through an electric cable and an attenuator to the data transmission A. Attenuation of long distance cable is simulated by attenuator. 1 PPS output from the data transmission A is regarded as a reference; bias between these two clocks is tested by TIC. PC takes note of the clock bias, which is used to produce a control signal according to the principle of FIR filter. The sampling interval is 10 seconds. 150 data pieces are chosen, and the trend terms after invariance in those data is removed are shown in Figure 6.

Devices are also used to design the clock calibration experiment that time signal is transmitted through radio channel. Layout is shown in Figure 7.

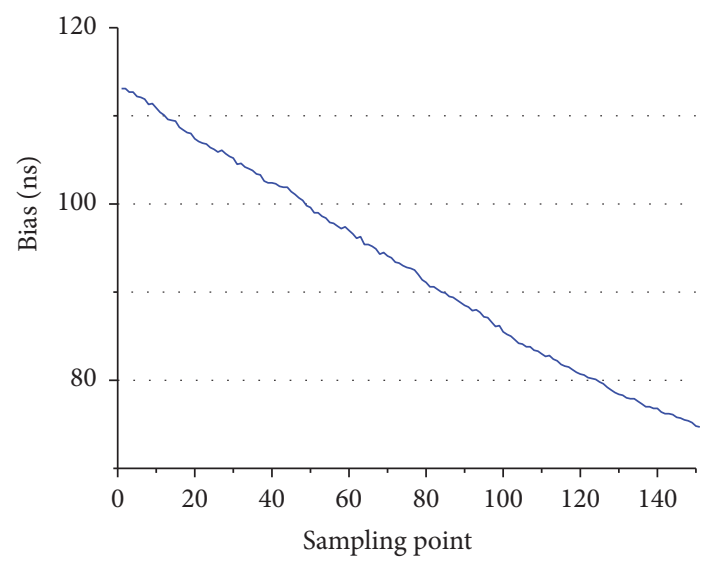

Figure 6: Clock bias acquired by wired channel.

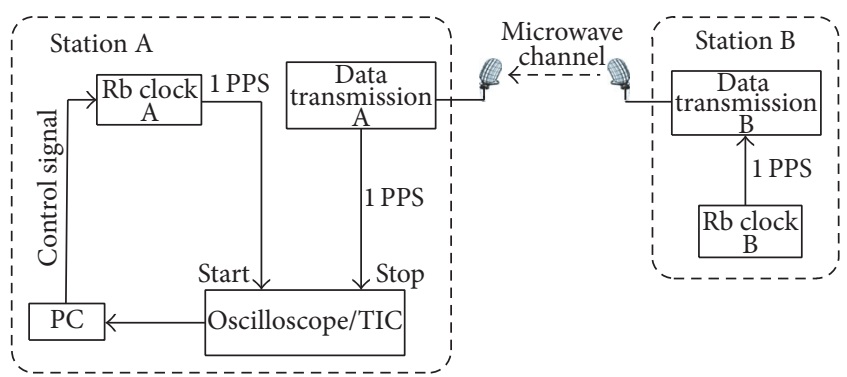

FIgURE 7: Clock calibration through radio channel.

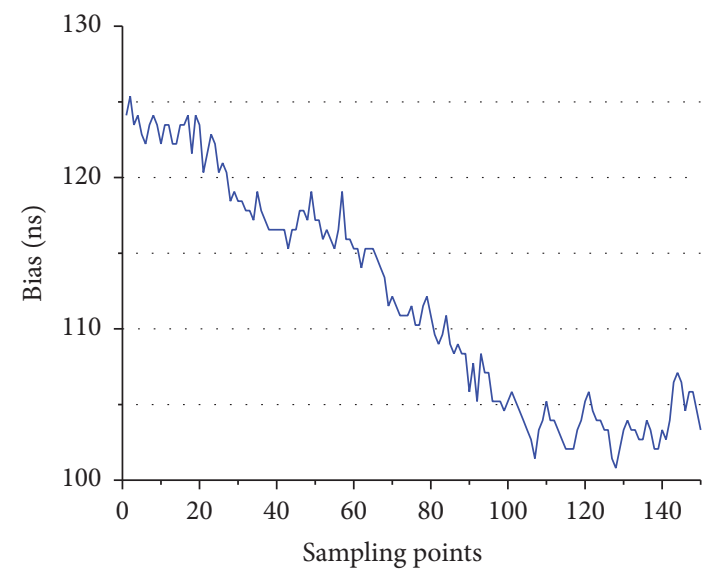

Figure 8: Clock bias acquired by radio channel.

The sampling interval of this experiment is also 10 seconds. Figure 8 shows the trend terms of these data.

As shown in Figures 6 and 8, the downward trend of bias indicates that these two clocks tend to be synchronized with calibration running. Noises in microwave channel and multipath effect bring jitter to the clock bias, which indicates that noises have negative effect on clock calibration.

4.2. Prediction Results. In order to get the accuracy of $\operatorname{GM}(1,1)$ model improved by IPSO, polynomial model, 
TABLE 1: Statistics of the different models (ns).

\begin{tabular}{|c|c|c|c|c|c|c|c|c|c|}
\hline \multirow{2}{*}{ Clock bias } & \multicolumn{3}{|c|}{ Polynomial model } & \multicolumn{3}{|c|}{$\operatorname{GM}(1,1)$ model } & \multicolumn{3}{|c|}{ IPSO-GM $(1,1)$ model } \\
\hline & $E_{\max }$ & ME & $\mathrm{SD}$ & $E_{\max }$ & $\mathrm{ME}$ & SD & $E_{\max }$ & $\mathrm{ME}$ & $\mathrm{SD}$ \\
\hline Data 1 & 1.543 & -0.414 & 0.641 & 0.822 & 0.383 & 0.325 & 0.544 & 0.127 & 0.301 \\
\hline Data 2 & 3.247 & -1.776 & 0.850 & 2.758 & -1.248 & 0.799 & 1.614 & -0.323 & 0.623 \\
\hline
\end{tabular}

GM $(1,1)$ model, and GM( 1,1$)$ improved by IPSO are, respectively, established by using 120 sets of data acquired in these two experiments; the last 30 sets of data are used to test these different models.

Individual atomic clocks have different frequency offset characteristic, the relationship between order of polynomial and atomic clock is defined as

$$
x(t)=a+b t+\beta \cdot \frac{c t^{2}}{2}, \quad \beta= \begin{cases}1, & \mathrm{Rb}, \\ 0, & \mathrm{Cs} .\end{cases}
$$

Considering that $\mathrm{Rb}$ atomic clock is used in these experiments, polynomial with one order is used. Figure 9 shows the prediction errors of different models.

In Figure 9, the clock bias acquired by wired channel and microwave channel is expressed as data 1 and data 2, respectively. Judgments are made in several aspects, such as maximum absolute error $\left(E_{\max }\right), \mathrm{ME}$, and standard error (SD). Table 1 describes the values of these judgments.

Figure 9 and Table 1 illustrate that the MEs of two results predicted through $\mathrm{GM}(1,1)$ model improved by IPSO are less than $0.5 \mathrm{~ns}$. Accuracy of ME increases by $66.4 \% \sim 76.7 \%$ compared to the traditional $\operatorname{GM}(1,1)$ model. Also, in terms of accuracy and stability, the improved model is superior to other models. Therefore, when a channel interruption or equipment failure happens, the bias predicted by $\operatorname{GM}(1,1)$ model improved by IPSO can be used to produce the control signal, which is used to synchronize the clock.

Also, the limitation of parameters selection range at the beginning can not only overcome the blindness in IPSO but also reduce the calculation time in a certain degree. The simulation process on PC platform indicates that running time of improved prediction model is less than $5 \mathrm{~s}$, which concludes that the improved prediction model can completely match the real-time requirement.

At the same time, the accuracy of all prediction models built using data 1 is better than using data 2 . The noises in clock bias have negative effect not only on performance of clock calibration, but also on the accuracy of clock bias prediction.

\section{Conclusion}

The failure to transfer time signal and equipment's breakdown will bring the failure to time synchronization system. Aiming at this problem, we parallel the clock bias system with clock calibration system. Also, $\operatorname{GM}(1,1)$ model improved by IPSO is proposed to predict the clock bias. Clock calibration experiments that time signal is transferred through wire and microwave channel are conducted. The parts of clock bias recorded in the process of the experiment are used to evaluate

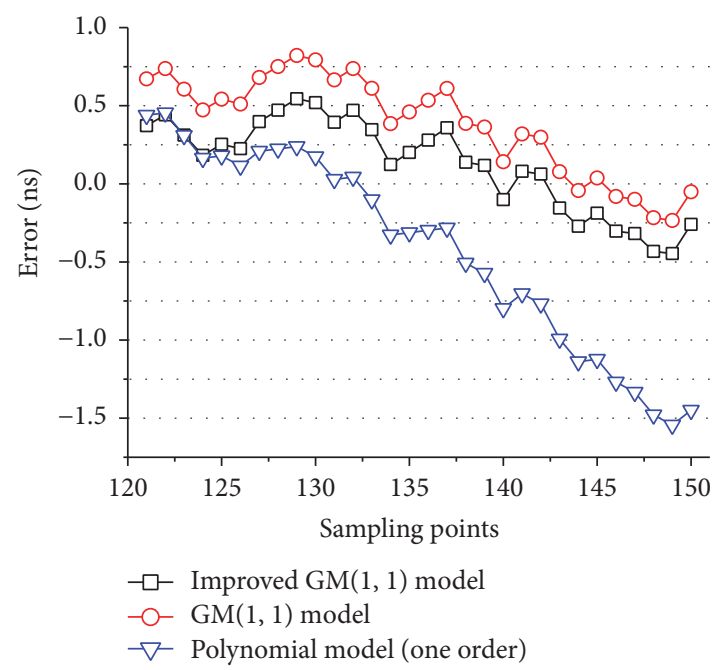

(a) Data 1

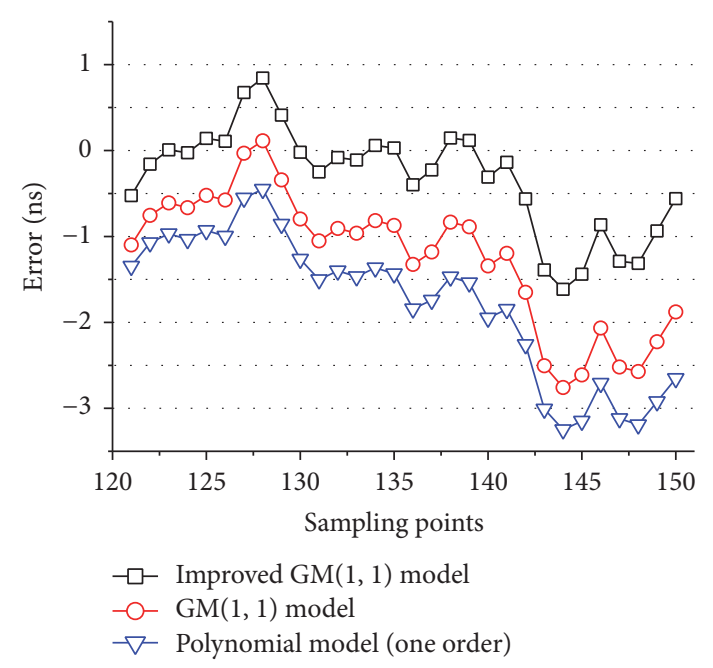

(b) Data 2

FiguRE 9: Errors of different prediction models.

this improved model. The consequence indicates that the $\mathrm{ME}$ of improved model is less than $0.5 \mathrm{~ns}$. Also, in view of accuracy and stability, the improved model is superior to other models. Therefore, when a failure happens, the clock bias predicted through $\operatorname{GM}(1,1)$ model improved by IPSO can be used to produce the control signal, which is vital to synchronize the clock.

\section{Competing Interests}

The authors declare that there is no conflict of interests regarding the publication of this paper. 


\section{Acknowledgments}

This work was supported by the National Natural Science Foundation of China under Grant no. 61571459. The authors also would like to thank IGS for granting access to satellite clock bias data.

\section{References}

[1] E. Mallada, X. Meng, M. Hack, L. Zhang, and A. Tang, "Skewless network clock synchronization without discontinuity: convergence and performance," IEEE/ACM Transactions on Networking, vol. 23, no. 5, pp. 1619-1633, 2015.

[2] M. R. Kosanovic and M. K. Stojcev, "RPATS-Reliable power aware time synchronization protocol," Microelectronics Reliability, vol. 54, no. 1, pp. 303-315, 2014.

[3] Z. Wang, L. Zhao, S. Wang, J. Zhang, B. Wang, and L. Wang, "COMPASS time synchronization and disseminationtoward centimetre positioning accuracy," Science China Physics, Mechanics \& Astronomy, vol. 57, no. 9, pp. 1788-1804, 2014.

[4] A. V. Makarenko, "Analysis of the time structure of synchronization in multidimensional chaotic systems," Journal of Experimental and Theoretical Physics, vol. 120, no. 5, pp. 912921, 2015.

[5] W. Xiang, B. Xu, W. Mou, and F. Wang, "A clock error calibration algorithm based on phase lock loop in GNSS time synchronization receiver," Journal of National University of Defense Technology, vol. 35, no. 2, pp. 115-119, 2013.

[6] Q. Liu, J. Sun, X. Chen, J. Liu, and Q. Zhang, "Analysis of two way troposphere time transfer and its delay errors," Acta Geodaetica et Cartographica Sinica, vol. 43, no. 4, pp. 341-347, 2014.

[7] M. Jabbarifar, M. Dagenais, and A. Shameli-Sendi, "Online incremental clock synchronization," Journal of Network and Systems Management, vol. 23, no. 4, pp. 1034-1066, 2015.

[8] S.-G. Lin, "Assisted adaptive extended Kalman filter for lowcost single-frequency GPS/SBAS kinematic positioning," GPS Solutions, vol. 19, no. 2, pp. 215-223, 2015.

[9] H. Xiong and D. Li, "Nuclear reactor doubling time calculation using FIR filter," Energy Procedia, vol. 39, pp. 3-11, 2013.

[10] L. Arceo-Miquel, Y. S. Shmaliy, and O. Ibarra-Manzano, "Optimal synchronization of local clocks by GPS 1PPS signals using predictive FIR filters," IEEE Transactions on Instrumentation and Measurement, vol. 58, no. 6, pp. 1833-1840, 2009.

[11] X. Cui and W. Jiao, "Grey system model for the satellite clock error predicting," Geomatics and Information Science of Wuhan University, vol. 30, no. 5, pp. 447-450, 2005.

[12] G. W. Huang, Q. Zhang, and G. C. Xu, "Real-time clock offset prediction with an improved model," GPS Solutions, vol. 18, no. 1, pp. 95-104, 2014.

[13] L. Wu, S. Liu, D. Chen, L. Yao, and W. Cui, "Using gray model with fractional order accumulation to predict gas emission," Natural Hazards, vol. 71, no. 3, pp. 2231-2236, 2014.

[14] H. P. Tserng, T. L. Ngo, P. C. Chen, and L. Quyen Tran, "A grey system theory-based default prediction model for construction firms," Computer-Aided Civil and Infrastructure Engineering, vol. 30, no. 2, pp. 120-134, 2015.

[15] A. B. Hashemi and M. R. Meybodi, "Cellular PSO: a PSO for dynamic environments," in Advances in Computation and Intelligence: 4th International Symposium, ISICA 2009 Huangshi, China, Ocotober 23-25, 2009 Proceedings, vol. 5821 of Lecture Notes in Computer Science, pp. 422-433, Springer, Berlin, Germany, 2009.
[16] B. Tran, B. Xue, and M. Zhang, "Improved PSO for feature selection on high-dimensional datasets," in Simulated Evolution and Learning, vol. 8886, pp. 912-921, Springer, 2014.

[17] C. Sudheer, R. Maheswaran, B. K. Panigrahi, and S. Mathur, "A hybrid SVM-PSO model for forecasting monthly streamflow," Neural Computing and Applications, vol. 24, no. 6, pp. 1381-1389, 2014. 


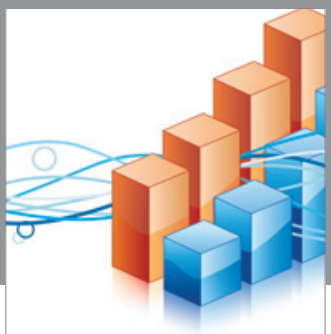

Advances in

Operations Research

vatem alat4

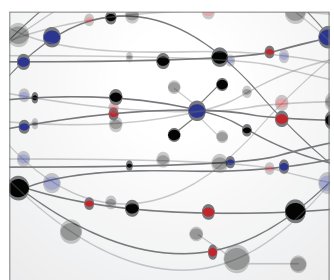

\section{The Scientific} World Journal
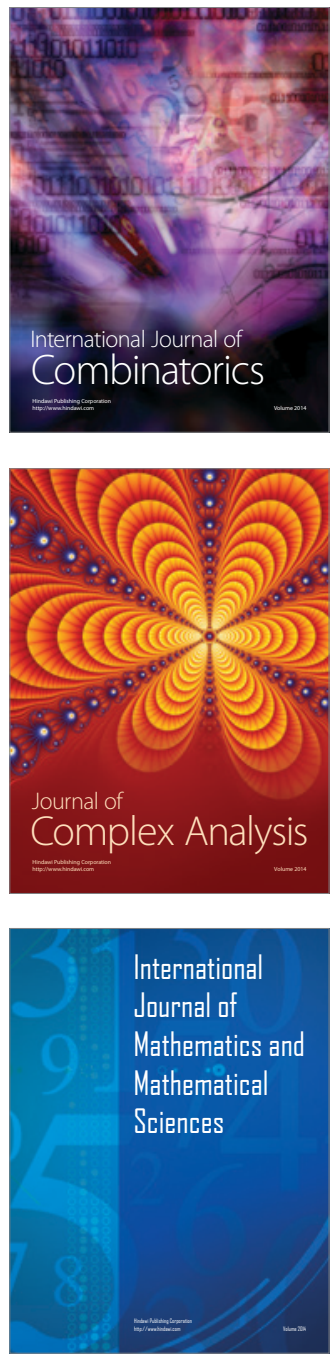
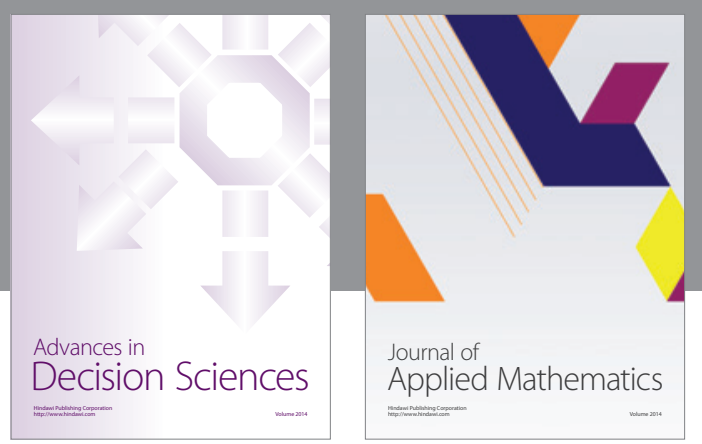

Algebra

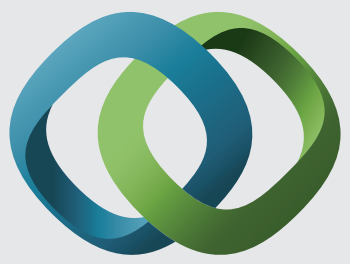

\section{Hindawi}

Submit your manuscripts at

http://www.hindawi.com
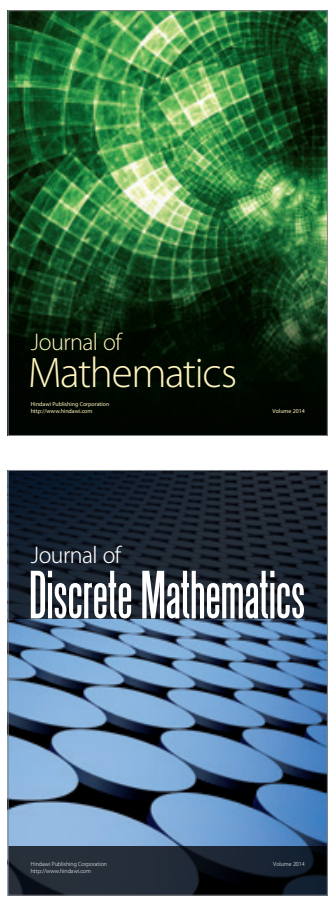

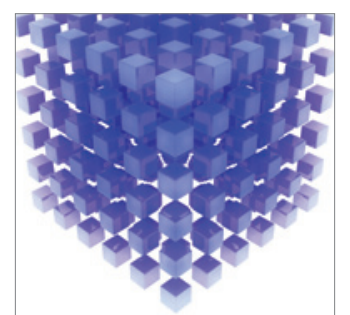

Mathematical Problems in Engineering
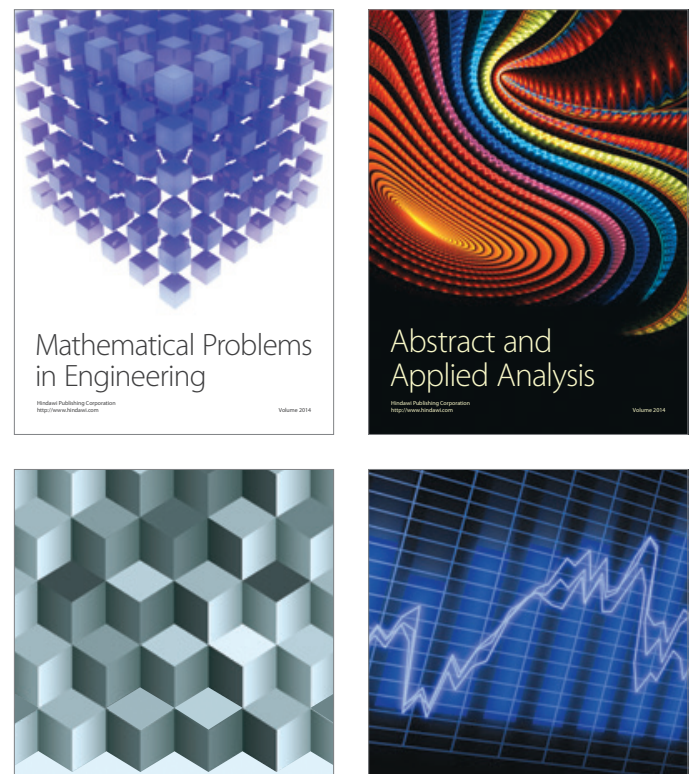

Journal of

Function Spaces

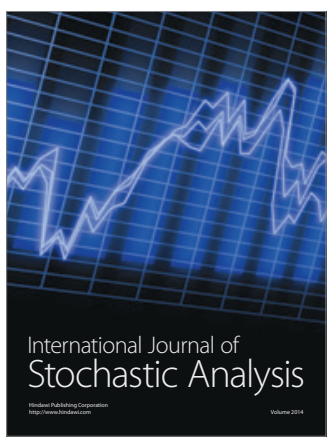

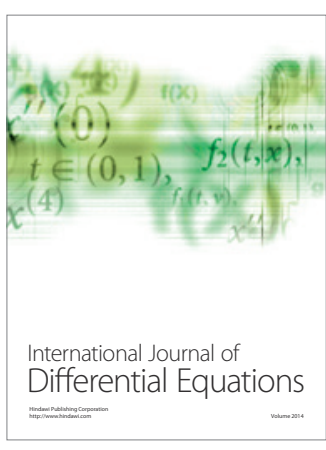
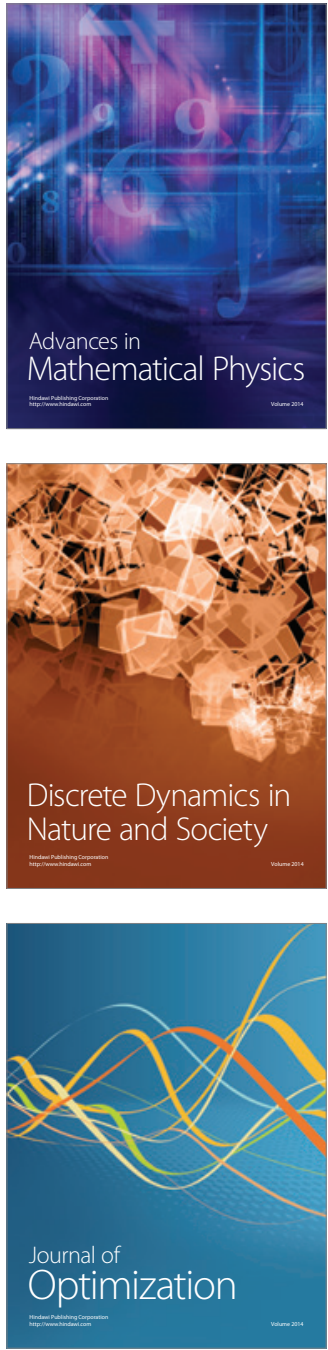